\title{
Étude multifactorielle de l'aptitude à l'effort chez le cheval de selle
}

\author{
E. Barrey, J.P. Valette et R. Wolter
}

Ecole nationale vétérinaire d'Alfort, laboratoire de physiologie sportive (INRA), 7, avenue du Général-de-Gaulle, 94704 Maisons-Alfort Cedex, France

(recu le 15 janvier 1989, accepté le 23 juin 1989)

\begin{abstract}
Résumé - La performance sportive, chez le cheval, résulte du concours de facteurs extrinsèques et intrinsèques, d'ordre physiologique, énergétique ou biomécanique. Cette étude tente de préciser les potentialités de 16 chevaux de selle par une analyse multifactorielle basée sur des critères cardiaques, hématologiques, énergétiques et sur des paramètres d'allure. L'analyse en composantes principales (ACP) décrit les liens et les redondances, ainsi que les antagonismes entre les variables étudiées. L'analyse factorielle discriminante (AFD) différencie les races en fonction de ces mêmes variables. Ces modalités d'analyse multifactorielle, qui réalisent la synthèse de toutes les informations disponibles, semblent bien adaptées à l'estimation des aptitudes sportives.
\end{abstract}

\footnotetext{
analyse multifactorielle - aptitudes sportives - test d'effort - paramètres d'allure critères de forme physique
}

Summary - Multicomponent analysis of exercise fitness in saddle horses. Equine exercise performance depends on numerous extrinsic factors which interact with each other. Physiological, energetic and biochemical factors have to be investigated simultaneously to study exercise performance capacity. The purpose of this study was to determine the capacities of 16 saddle horses by measuring cardiac, haematological, energetic and gait parameters. Multivariate statistical methods such as principal component analysis and discriminant analysis were used to describe the relationships between variables and to compare the exercise fitness of each horse, taking into account all the information obtained. These statistical methods indicated interesting relations of causality or redundancy between the parameters. Study of the horses revealed a better aptitude in sprint, resistance or endurance exercise in some of them. Discriminant analysis distinguished between breeds on the basis of the different physiological and gait parameters measured. It was concluded that multivariate analysis was well adapted to analyse the exercise fitness on the basis of several criteria by providing synthetic information.

multicomponent analysis - exercise fitness - exercise testing - gait parameters - fitness criteria 


\section{INTRODUCTION}

La performance sportive résulte d'un ensemble de facteurs extrinsèques et intrinsèques au cheval. Les conditions météorologiques, les qualités mécaniques de la piste, le rationnement alimentaire et la technique du cavalier font partie des facteurs extrinsèques (Pratt, 1984; Wolter, 1987; Frape, 1988). Parmi les facteurs intrinsèques qui sont influencés par l'entraînement, les caractéristiques physiologiques, énergétiques et biomécaniques du cheval ont fait l'objet de nombreux travaux cités par Persson (1983), McMiken (1983), Thornton (1985), Leach \& Dagg (1983) et Dalin \& Jeffcott (1985).

L'adaptation physiologique à l'exercice sportif fait intervenir des facteurs cardiaques, circulatoires, respiratoires, hématologiques, biochimiques et musculaires déjà bien étudiés indépendamment les uns des autres. Cependant, d'autres critères physiologiques seraient à prendre en compte pour comprendre la performance ou la contre-performance sportive d'un cheval, notamment des critères neurologiques, psychologiques, énergétiques et biomécaniques.

Sur le plan énergétique, les modalités de production de l'énergie métabolique, déjà bien connues, sont encore très mal reliées aux données biomécaniques de l'exercice. Or, d'après les travaux effectués chez le sportif humain, les notions d'efficacité mécanique du mouvement et de rendement énergétique semblent déterminants pour parvenir à une bonne performance sportive (Cavanagh \& Kram, 1985; Daniels, 1985).

Jusqu'à présent, peu de travaux de recherche ont essayé d'étudier les rapports entre l'adaptation physiologique, le métabolisme énergétique et la biomécanique des allures. Persson et al. (1983), Wilson et al. (1983), Valberg et al. (1985), Pagan \& Hintz (1986a et b), Thiel et al. (1987), Thornton et al. (1987) et Valette \& Wolter (1987) ont mis en évidence des relations entre certains paramètres physiologiques cardiaques, respiratoires et biochimiques. Hoyt \& Taylor (1981) ont montré l'existence, pour chaque allure, d'une vitesse optimale pour laquelle la dépense énergétique est minimale. Hörnicke et al. (1983) et Attenburrow (1983) ont étudié le couplage entre la fréquence des foulées et la fréquence respiratoire au trot et au galop.

Une des difficultés de l'étude multifactorielle de l'aptitude sportive réside dans le choix de la méthode d'analyse des résultats. Les méthodes statistiques classiques, qui comparent les variables deux à deux, sont insuffisantes pour effectuer une synthèse de la totalité des informations obtenues. Le recours aux méthodes d'analyse factorielle, telles que l'analyse en composantes principales (ACP) et une variante, l'analyse factorielle discriminante (AFD), semble bien adapté à l'étude des phénomènes biologiques où la variabilité individuelle est de règle (Gutteridge et al., 1979; Silver et al., 1983; Valette \& Wolter, 1988). Ces outils statistiques puissants décrivent les relations entre les variables et permettent aussi de situer les individus les uns par rapport aux autres, en tenant compte simultanément de toutes les informations recueillies.

L'objet de ce travail est de montrer l'intérêt des méthodes d'analyses multivariées pour comparer l'aptitude sportive de chevaux de selle grâce à la mesure de 3 catégories de paramètres déjà bien connus : des paramètres physiologiques, énergétiques et locomoteurs. Ainsi, la première catégorie décrit le fonctionnement des organes qui concourent à l'approvisionnement énergétique des muscles; la deuxième précise le type de métabolisme énergétique mis en jeu au cours de l'effort; 
enfin, la troisième analyse la mécanique d'allure adoptée par le cheval.

\section{MATÉRIEL ET MÉTHODES}

\author{
Animaux \\ Onze chevaux de race Selle Français, 2 de race \\ Trotteur, 2 de race Pur-Sang et 1 Poney appar- \\ tenant à la Société hippique de l'école vétéri- \\ naire d'Alfort (SHEVA) ont été testés par \\ groupes de 2 dans un manège couvert de $40 x$ \\ $19 \mathrm{~m}$. Leurs caractéristiques de format et de \\ race sont données dans le Tableau I. Tous les \\ chevaux étaient en bonne santé au moment des \\ épreuves et travaillaient en moyenne $10 \mathrm{~h}$ par \\ semaine.
}

\section{Test d'aptitude physique}

L'ensemble des chevaux a été testé durant la période d'automne. Chaque semaine, 2 chevaux sont testés toujours le même jour et aux mêmes heures.

Après un échauffement d'une quinzaine de minutes au pas, les chevaux montés effectuent 5 épreuves successives de 5 min chacune, entrecoupées de périodes de repos de $3 \mathrm{~min}$.

Le test comprend 5 paliers d'allures et de vitesses moyennes croissantes : pas (1,5 $2 \mathrm{~m} / \mathrm{s})$, petit trot $(2,7-3,2 \mathrm{~m} / \mathrm{s})$, trot allongé $(3,8-4,7 \mathrm{~m} / \mathrm{s})$, petit galop $(4,9-6 \mathrm{~m} / \mathrm{s})$, galop allongé $(6,2-7,9 \mathrm{~m} / \mathrm{s})$. Au cours de ces paliers, le cavalier maintient le cheval sur une trajectoire rectangulaire de distance constante. Les cavaliers ont pour consigne d'intervenir le moins possible et de maintenir une allure constante pour chaque palier d'effort. La vitesse moyenne au tour de chaque cheval est mesurée par un chronométreur.

\section{Critères mesurés}

Au temps initial et immédiatement après chaque palier d'allure, un prélèvement sanguin sert aux déterminations suivantes :

- hématocrite;

- acide lactique, après déprotéinisation du sang au $1 / 3$ avec l'acide perchlorique $6 \mathrm{~N}$, et mesure à l'électrode spécifique (Analox).

Pendant toute la durée du test, la fréquence cardiaque (FC) est enregistrée toutes les $30 \mathrm{~s}$ au moyen d'un cardiofréquence-mètre (PEH 100 de Polar Electric). La lecture de l'enregistrement des fréquences cardiaques permet de calculer une moyenne de la FC pour chaque palier.

Les chevaux sont filmés à l'aide d'une caméra vidéo à chaque palier, entre 2 repères distants de $10 \mathrm{~m}$. L'analyse du film, image par image, sur un écran vidéo permet de mesurer manuellement les paramètres d'allure suivants pour chaque palier : la longueur moyenne d'une foulée (un cycle locomoteur) et la fréquence moyenne des foulées (inverse de la durée d'un cycle locomoteur).

\section{Paramètres synthétiques estimés}

L'étude de la relation entre la lactatémie et la vitesse sur l'ensemble des chevaux testés montre un décrochement de la courbe lactatémie - vitesse entre l'avant-dernier et le dernier palier a ${ }_{v i}=4,65 \mathrm{~m} / \mathrm{s}$ (Fig. 1). Cette vitesse correspond à l'intersection des 2 droites de régression lactatémie - vitesse obtenues à partir des points experimentaux, d'une part pour les paliers 1, 2, 3 et 4 et d'autre part pour les paliers 4 et 5 .

Cette vitesse sert de référence à la définition des différents paramètres décrits au Tableau I.

Pour effectuer une analyse globale des résultats obtenus à chaque palier, il a été choisi de calculer des paramètres synthétiques pour décrire l'aptitude sportive des chevaux. On distingue 3 catégories de paramètres (Tableau II) :

- une première catégorie de paramètres précise la capacité de travail du cheval en aérobie : $V_{150}, F_{v i}, P F C, H T_{v i}$ et PHT; 
Tableau I. Paramètres synthétiques calculés et caractéristiques de format des 16 chevaux étudiés.

$\begin{array}{llllllllll}\text { Paramètres } V_{150} & F C_{v i} & P F C & H T_{v i} & P H T & V_{4} & L a_{v i} & F E_{v i} & L F_{v i} & \text { Taille Poids Race }\end{array}$ $N^{\circ}$ du cheval

\begin{tabular}{|c|c|c|c|c|c|c|c|c|c|c|c|c|}
\hline 1 & 5,9 & 124 & 20,9 & 31,4 & 3,7 & 8,7 & 0,18 & 1,43 & 2,98 & 1,61 & 560 & TF \\
\hline 2 & 5,4 & 136 & 28,9 & 38,1 & 3,5 & 9,9 & 0,38 & 1,41 & 3,10 & 1,63 & 490 & TF \\
\hline 3 & 7,1 & 107 & 16,2 & 42,6 & 2,7 & 10,4 & 0,33 & 1,51 & 2,57 & 1,63 & 471 & SF \\
\hline 4 & 6,7 & 109 & 17 & 36,4 & 1,6 & 9 & 0,92 & 1,46 & 2,83 & 1,63 & 557 & SF \\
\hline 5 & 5,6 & 128 & 22,2 & 42,8 & 2,3 & 5,9 & 1,29 & 1,55 & 2,52 & 1,61 & 495 & SF \\
\hline 6 & 6,3 & 116 & 18,8 & 40,3 & 2,1 & 7,2 & 2,28 & 1,60 & 3,43 & 1,61 & 495 & SF \\
\hline 7 & 6,2 & 127 & 17,8 & 43,7 & 2,1 & 9 & 0,77 & 1,42 & 3,69 & 1,61 & 551 & SF \\
\hline 8 & 5,2 & 138 & 18,7 & 43,8 & 2,2 & 13,8 & 0,54 & 1,46 & 3,33 & 1,54 & 482 & PS \\
\hline 9 & 7,1 & 113 & 13,2 & 37,7 & 1 & 14 & 0,17 & 1,52 & 3,06 & 1,60 & 420 & PS \\
\hline 10 & 7,3 & 105 & 15,1 & 39,6 & 1 & 11,7 & 0,60 & 1,45 & 3,27 & 1,72 & 644 & SF \\
\hline 11 & 6,2 & 122 & 18,6 & 46,8 & 2,2 & 7,9 & 0,27 & 1,60 & 3,11 & 1,57 & 456 & SF \\
\hline 12 & 6 & 123 & 21,3 & 40,5 & 1,9 & 7,4 & 0,37 & 1,53 & 3,09 & 1,47 & 409 & Po \\
\hline 13 & 6,4 & 125 & 18,9 & 47,8 & 1,7 & 10,8 & 0,52 & 1,47 & 3,27 & 1,57 & 445 & SF \\
\hline 14 & 6,3 & 121 & 19 & 36,6 & 2,2 & 8,2 & 0,55 & 1,61 & 3,10 & 1,64 & 499 & SF \\
\hline 15 & 6,3 & 121 & 19 & 39,3 & 2,7 & 7,6 & 0,80 & 1,54 & 3,17 & 1,66 & 526 & SF \\
\hline 16 & 6,3 & 121 & 19 & 36,8 & 1,3 & 8,4 & 0,68 & 1,45 & 3,23 & 1,62 & 576 & SF \\
\hline
\end{tabular}

$v_{i}=4,65 \mathrm{~m} / \mathrm{s}: 279 \mathrm{~m} / \mathrm{min}: 16,7 \mathrm{~km} / \mathrm{h}$. 


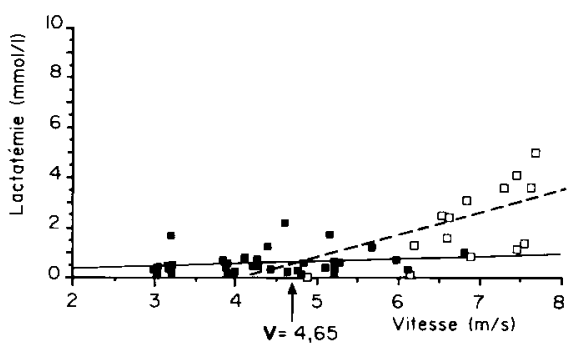

Fig. 1. Détermination de la vitesse de référence d'après la relation lactatémie-vitesse.

- une deuxième catégorie de paramètres explore le recrutement du métabolisme anaérobie pour un niveau d'effort donné : $V_{4}$ et $L a_{v i}$;

- une troisième catégorie de paramètres précise le type de mécanique d'allure adoptée par le cheval à une vitesse donnée : $F F_{v i}$ et $L F_{v i}$.

\section{Méthodes statistiques}

A partir des données mesurées à chaque palier pour chaque individu, les paramètres synthétiques sont interpolés grâce à des équations de régression. Les relations fréquence cardiaque - vitesse, hématocrite - vitesse et longueur des foulées - vitesse sont étudiées par des régressions linéaires, conformément aux travaux antérieurs de Persson (1967) et Leach (1987).

En revanche, la relation lactatémie - vitesse est analysée par une régression linéaire après transformation de la lactatémie en son logarithme népérien. Enfin, la relation fréquence des foulées - vitesse est étudiée grâce à une régression non linéaire du type :

$$
F F=F F \max \left(1-K e^{-c . v i t}\right)
$$

où FFmax représente la fréquence des foulées maximales que peut atteindre le cheval, $k$ et $c$ sont des coefficients constants déterminés pour chaque individu (Leach, 1987; Barrey, 1988).

Tableau II. Définition des critères d'aptitude à l'effort.

\begin{tabular}{|c|c|c|}
\hline Critères & Définitions & Unités \\
\hline$V_{150}$ & $\begin{array}{l}\text { La vitesse qui induit une fréquence } \\
\text { cardiaque de } 150 \text { batt/min }\end{array}$ & $\mathrm{m} / \mathrm{s}$ \\
\hline$F C_{v i}$ & $\begin{array}{l}\text { La fréquence cardiaque mesurée pour la } \\
\text { vitesse } \mathrm{Vi}\end{array}$ & batt/min \\
\hline PFC & $\begin{array}{l}\text { La pente de la droite de régression de la } \\
\text { relation FC - vitesse }\end{array}$ & batt. $\mathrm{min}^{-1} / \mathrm{m} \cdot \mathrm{s}^{-1}$ \\
\hline$H T_{v i}$ & L'hématocrite mesuré pour la vitesse vi & $\%$ \\
\hline PHT & $\begin{array}{l}\text { La pente de la droite de régression de la } \\
\text { relation HT - vitesse }\end{array}$ & $\% / m \cdot s^{-1}$ \\
\hline$V_{4}$ & $\begin{array}{l}\text { La vitesse à laquelle la lactémie atteint } \\
4 \mathrm{mmol} / \mathrm{l}\end{array}$ & $\mathrm{m} / \mathrm{s}$ \\
\hline $\mathrm{La}_{\mathrm{vi}}$ & La lactémie mesurée pour la vitesse $v i$ & $\mathrm{mmol} /$ \\
\hline$F F_{v i}$ & $\begin{array}{l}\text { La fréquence des foulées mesurée pour la } \\
\text { vitesse }_{\text {vi }}\end{array}$ & foulées/s ou Hz \\
\hline $\mathrm{LF}_{\mathrm{vi}}$ & $\begin{array}{l}\text { La longueur des foulées mesurée pour la } \\
\text { vitesse } \\
\text { vi }\end{array}$ & $m$ \\
\hline
\end{tabular}


Pour chaque cheval, il est ainsi calculé 8 paramètres synthétiques qui constituent 8 variables quantitatives. Le tableau de résultats individus $x$ variables quantitatives est analysé à l'aide de l'ACP. Les différences d'aptitude à l'effort liées à la race des chevaux seront mises en évidence à l'aide de l'AFD.

L'ACP permet d'étudier simultanément les rapports entre les variables et de comparer les individus entre eux (Foucart, 1985). De manière schématique, le principe de l'ACP consiste à créer $n$ ( $n=$ nombre de variables étudiées) nouvelles variables (appelées composantes principales nos $1,2,3, \ldots, n$ ), qui résultent d'une combinaison linéaire des variables étudiées et dont la propriété est d'être indépendantes. Ainsi, chaque variable et chaque individu est caractérisé par $n$ valeurs de composantes principales. La numérotation des composantes principales reflète leur importance dans la description de la variabilité totale. Les 2 premières composantes constituent donc le couple qui explique la plus grande part de la variation totale. II s'ensuit que les résultats de l'ACP peuvent être représentés graphiquement par la projection des variables ou des individus dans le plan défini par les 2 premières composantes. Ce plan regroupe la plus grande part de l'information et est le plus souvent suffisant pour décrire les résultats. Si la troisième composante représente une part importante de la variation totale, une représentation dans l'espace devient nécessaire pour décrire les données. L'AFD est une ACP particulière, effectuée sur les moyennes des groupes définis à l'aide d'une variable qualitative. Cette dernière méthode tente de différencier les groupes en tenant compte de la totalité des variables étudiées. Pour plus de détails sur la theorie de ces méthodes statistiques élaborées, il convient de se reporter à l'ouvrage de Foucart (1985).

Pour exposer les résultats des 2 analyses factorielles, nous nous appuierons sur les règles d'interprétation suivantes des représentations graphiques :

- plus une variable ou un individu sont éloignés de l'origine des axes, mieux ils sont représentés dans le plan considéré;

-2 variables sont corrélées positivement ou 2 individus se ressemblent, pour autant qu'ils sont proches l'un de l'autre dans l'espace. Deux variables sont corrélées négativement ou 2 individus sont très différents l'un de l'autre, pour au- tant qu'ils sont diamétralement opposés par rapport à l'origine des axes.

\section{RÉSULTATS}

L'ensemble des paramètres synthétiques calculés est présenté dans le Tableau II. Celui-ci comprend, pour les 16 individus, 11 variables quantitatives et 1 variable qualitative (la race). L'ACP est pratiquée sur les valeurs centrées et réduites de $\$ 1$ variables quantitatives. L'AFD est effectuée en adjoignant aux variables quantitatives la variable qualitative définie par la race.

\section{Résultats de I'ACP}

Dans l'analyse des résultats, nous prendrons en compte les 4 premières composantes, qui, globalement, expliquent respectivement $31,7,19,5,17,1$ et $12,8 \%$ de la variation totale. Le plan de représentation $1 \times 2$ recouvre $51,2 \%$ de la variation totale.

\section{Étude des variables}

La majorité des variables est bien représentée dans le plan $1 \times 2$ (Fig. 2), excepté la lactatémie (La), la fréquence des foulées (FF), la longueur des foulées (LF) ainsi que la vitesse $\mathrm{V}_{4}$.

Les variables $F F, V_{4}$ et $L a$ sont bien représentées dans le plan $1 \times 3$, LF dans le plan $1 \times 4$ (plans non figurés).

Du fait des corrélations existantes entre les variables et les composantes princi- 


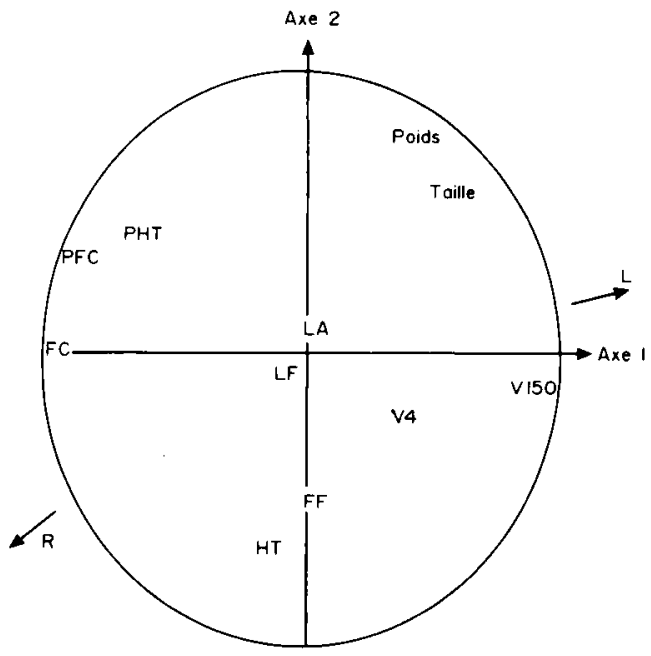

Fig. 2. Représentation des corrélations des variables dans le plan $1 \times 2$. Elle met en évidence l'importance des paramètres cardio-vasculaires (V150, FC, PFC, PHT, HT) pour l'aptitude à l'effort. Ces facteurs définissent, en effet, les 2 premiers axes de I'ACP. Les flèches indiquent l'orientation de 2 types d'aptitude à l'effort différents : l'effort bref et rapide (R) et l'effort long (L). pales (Tableau III), il est possible d'attribuer une signification biologique à chacun des axes 1, 2, 3 et 4 :

- l'axe 1 caractérise la réponse du système cardio-vasculaire à l'effort imposé $\left(\mathrm{V}_{150}, \mathrm{FC}, \mathrm{PFC}, \mathrm{PHT}\right)$;

- l'axe 2 indique l'aptitude à mobiliser le "pool" de globules rouges stockés dans la rate;

- l'axe 3 précise le niveau de recrutement du métabolisme anaérobie, en liaison avec la fréquence des foulées adoptées par le cheval à 4,65 m.s ${ }^{-1}$;

- l'axe 4 caractérise l'allongement de la foulée pour le niveau d'effort défini.

Le tableau des corrélations (Tableau IV), qui se traduit en grande partie par la représentation des variables dans le plan $1 \times 2$ (Fig. 2), révèle les rapports entre celles-ci. Ainsi, $V_{150}$ et FC sont exactement antagonistes et apportent donc le même type d'information $\left(r_{14 d d l}=-0,95\right)$. PFC est bien corrélée avec PHT ( $r_{14 d d l}=$ $0,69)$, avec $V_{150}\left(r_{14 d d l}=0,77\right)$ et avec FC $\left(r_{14 d d}=0,71\right)$.

Tableau III. Corrélations entre les variables et les axes.

Composantes principales

Variables

Axe 1

Axe 2

Axe 3

Axe 4

$\begin{array}{lcccr}V_{150} & 0,937 & -0,109 & 0,074 & 0,162 \\ \text { FC } & -0,905 & 0,014 & -0,268 & -0,211 \\ \text { PFC } & -0,851 & 0,352 & 0,0945 & 0,047 \\ \text { HT } & -0,139 & -0,675 & -0,051 & -0,326 \\ \text { PHT } & -0,684 & 0,42 & 0,114 & 0,297 \\ V_{4} & 0,321 & -0,198 & -0,811 & 0,069 \\ \text { La } & 0,021 & 0,069 & 0,659 & -0,609 \\ \text { FF } & 0,037 & -0,512 & 0,737 & 0,05 \\ \text { FL } & -0,0045 & -0,034 & -0,361 & -0,813 \\ \text { Taille } & 0,559 & 0,656 & 0,131 & -0,06 \\ \text { Poids } & 0,404 & 0,797 & -0,038 & -0,312\end{array}$


Bien que les corrélations ne soient pas significatives au seuil de $5 \%$, certaines relations sont intéressantes à souligner. $V_{4}$ et La sont relativement antagonistes l'une de lautre et ne recouvrent d'ailleurs pas exactement la même signification biologique. II faut également noter la plus forte liaison entre les variables La et FF qu'entre $L a$ et LF.

Les variables fréquence des foulées (FF) et longueur des foulées (LF) décrivent bien des composantes distinctes de l'allure : cadence et allongement. Ainsi, l'axe 3 de I'ACP prend en compte essentiellement la cadence (FF), tandis que l'axe 4 recouvre uniquement l'amplitude des foulées (LF).

II n'existe pas de liaison entre l'amplitude des foulées (LF) et la taille du cheval. En revanche, plus le cheval est lourd, plus la cadence de l'allure qu'il adopte semble lente (FF basse) [corrélation non significative au seuil de 5\%]. Enfin, la forte liaison des variables masse et taille $\left(r_{14 \mathrm{ddl}}=0,74\right)$ provient de l'harmonie du format des chevaux de l'échantillon.

\section{Etude des individus}

La Figure 3 donne la projection des résultats de l'ACP sur le plan $1 \times 2$ pour l'ensemble des individus.

La plupart des individus sont correctement représentés dans le plan $1 \times 2$, excepté les individus nos 6,7 et 14 . Les $n^{\text {os }} 6$ et 7 sont mieux caractérisés dans les plans $1 \times 3$ et $1 \times 4$, le $n^{\circ} 14$ dans le plan $1 \times 3$. Graphiquement, la mauvaise représentation des individus se traduit par leur proximité de l'intersection des axes des composantes principales (Fig. 3).

D'après les représentations des individus dans les plans $1 \times 2$, certains chevaux se révèlent avoir une aptitude sportive particulière. Les individus $n^{\text {os }} 5,6,8$ et 12 sont regroupés sur la gauche de la Figure 3 ; ils ont une aptitude à l'effort semblable. Alors que les individus nos $3,4,9,10$ et 16 se situent sur la droite de la Figure 3 et présentent une aptitude à l'effort différente du groupe de gauche. Deux individus ( $n^{\text {os }}$ 1 et 2) apparaissent avoir très peu d'apti-

Tableau IV. Corrélations entre les variables étudiées à l'aide de l'ACP $(n=16)$.

\begin{tabular}{|c|c|c|c|c|c|c|c|c|c|c|c|}
\hline & $V_{150}$ & $F C_{v i}$ & $P F C$ & $H T_{v i}$ & $P H T$ & $V_{4}$ & $L a_{v i}$ & $F F_{v i}$ & $L F_{v i}$ & Taille & Poids \\
\hline$V_{150}$ & 1,00 & & & & & & & & & & \\
\hline$F C_{v i}$ & $-0,95$ & 1,00 & & & & & & & & & \\
\hline $\begin{array}{l}P F C \\
H T_{v i}\end{array}$ & $\begin{array}{l}-0,77 \\
-0,07\end{array}$ & $\begin{array}{l}\mathbf{0 , 7 1} \\
0,20\end{array}$ & $\begin{array}{r}1,00 \\
-0,09\end{array}$ & 1,00 & & & & & & & \\
\hline $\begin{array}{l}P H T \\
V_{4}\end{array}$ & $\begin{array}{r}-0,58 \\
0,23\end{array}$ & $\begin{array}{r}0,50 \\
-0,06\end{array}$ & $\begin{array}{r}\mathbf{0 , 6 9} \\
-0,42\end{array}$ & $\begin{array}{r}-0,20 \\
0,08\end{array}$ & $\begin{array}{r}1,00 \\
-0,32\end{array}$ & 1,00 & & & & & \\
\hline$L a_{v i}$ & $-0,06$ & $-0,08$ & $-0,04$ & 0,07 & $-0,09$ & $-0,46$ & 1,00 & & & & \\
\hline$F F_{v i}$ & 0,11 & $-0,20$ & $-0,17$ & 0,20 & $-0,10$ & $-0,41$ & 0,34 & 1,00 & & & \\
\hline$L F_{v i}$ & $-0,10$ & 0,24 & $-0,09$ & 0,17 & $-0,21$ & 0,20 & 0,16 & $-0,19$ & 1,00 & & \\
\hline $\begin{array}{l}\text { Taille } \\
\text { Poids }\end{array}$ & $\begin{array}{l}0,46 \\
0,20\end{array}$ & $\begin{array}{l}-0,46 \\
-0,27\end{array}$ & $\begin{array}{l}-0,19 \\
-0,12\end{array}$ & $\begin{array}{l}-0,34 \\
-0,41\end{array}$ & $\begin{array}{l}-0,04 \\
-0,07\end{array}$ & $\begin{array}{r}0,03 \\
-0,07\end{array}$ & $\begin{array}{l}0,15 \\
0,17\end{array}$ & $\begin{array}{l}-0,14 \\
-0,44\end{array}$ & $\begin{array}{r}-0,06 \\
0,18\end{array}$ & $\begin{array}{l}1,00 \\
\mathbf{0 , 7 4}\end{array}$ & 1,00 \\
\hline
\end{tabular}

En gras, corrélations significatives au seuil de $5 \%$. 
tude à effectuer un effort. Les autres individus ( $n^{\circ \mathrm{s}} 7,11,13,14$ et 15 ), placés en position moyenne de l'axe 1 , ont des potentialités intermédiaires, sans avoir d'aptitude sportive bien affirmée.

\section{Résultats de l'AFD}

\section{Etude des variables}

L'axe 1 de l'AFD recouvre schématiquement les axes 1, 2 et 3 de l'ACP, si bien que le plan $1 \times 2$ explique $72 \%$ de la variation totale. Les axes 1,2 et 3 expliquent respectivement $36,9,35,1$ et $28 \%$ de la variation totale.

L'axe 1 regroupe tous les paramètres physiologiques, l'axe 2 les paramètres d'allure et l'axe 3 les paramètres de format. Les paramètres physiologiques, réunis sur l'axe 1 , sont les plus aptes à discriminer les races.

\section{Etude des groupes (races)}

La Figure 4 et le Tableau $V$ des distances de Mahalanobis (Foucart, 1985) mettent en évidence les réponses physiologiques à l'effort propres à chaque race. Le Poney (groupe 4) se distingue très nettement des chevaux, avec une distance par rapport aux autres races $>3,69$. Parmi les chevaux, les 2 races les plus différentes sont les Pur-Sang (PS) et le Trotteur Français (TF), distantes de 3,43 et discriminés essentiellement par la mécanique d'allure (LF ef $F F$ ) ainsi que par la vitesse $V_{4}$, selon l'axe 2. La race Selle Français (SF) a une position intermédiaire entre TF et PS et se distinguent surtout par les paramètres physiologiques ( $\left.F C, V_{150}, P H T, L a\right)$ selon l'axe 1. Le cheval ayant un statut d'Autre Que Pur-Sang (AQPS), initialement classé parmi les SF, a été classé par l'AFD parmi le groupe de PS. Globalement, l'AFD parvient à un pourcentage d'individus bien classés de $94 \%$. Bien que les effectifs par

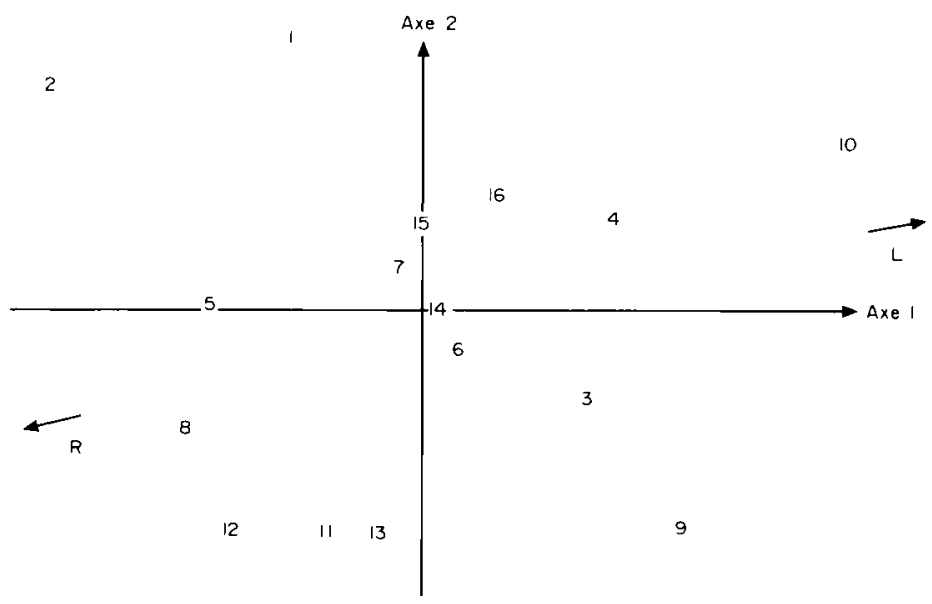

Fig. 3. Représentation graphique des individus dans le plan $1 \times 2$ de l'ACP. Les chevaux $5,6,8$ et 12 apparaissent mieux adaptés à soutenir un effort de sprint que les autres. Comparativement, les chevaux $3,4,9,10$ et 16 sont plus aptes à courir sur une longue distance. Les chevaux 3 et 9 ont davantage de résistance, tandis que les chevaux 4,10 et 16 sont plutôt endurants. 
race soient faibles, les critères mesurés (mêmes variables que pour l'ACP + variable qualitative race) semblent donc pouvoir très bien discriminer les races selon leur aptitude à l'effort.

\section{DISCUSSION}

L'existence, pour un paramètre donné, d'une seule valeur par individu exclut toute possibilité de recourir aux tests paramétriques classiques qui nécessitent l'hypothèse de normalité des distributions pour distinguer les animaux entre eux. La

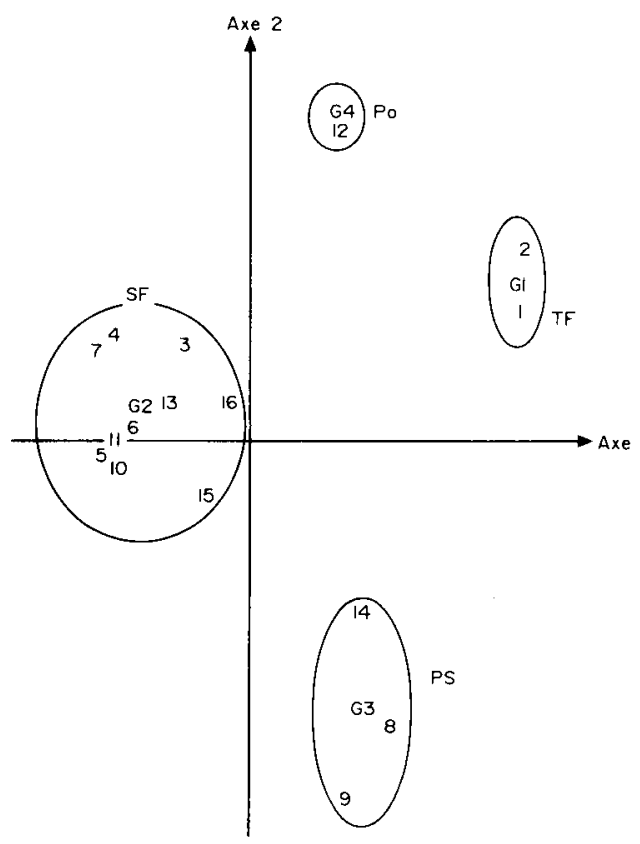

Fig. 4. Représentation graphique du plan $1 \times 2$ de l'AFD sur la réponse à l'effort propre à chaque race. Les notations employées sont : TF pour Trotteur Français, SF pour Selle Français, PS pour Pur-Sang, Po pour Poney; G1, G2, G3, G4 représentent les centres de gravité de chaque groupe de race. supériorité de l'analyse factorielle (ACP ou AFD) réside dans le fait qu'elle prend en compte toutes les variables et fait abstraction de la notion de normalité. Elle permet de discriminer au maximum variables et individus, même si les variations sont minimes et les effectifs faibles, ce que ne peuvent faire les tests classiques.

Toutefois, si elle révèle les ressemblances entre les variables étudiées (par le coefficient de corrélation), elle ne classe les individus que de manière relative par rapport au groupe et au test d'effort considéré. Rappelons qu'il s'agit d'une étude comparative sur un échantillon restreint de chevaux de selle : aussi faut-il considérer les aptitudes sportives prises dans un sens relatif. La confrontation des résultats graphiques portant sur les variables et les individus permet de définir, selon l'axe 1, 2 orientations particulières correspondant à des aptitudes sportives différentes. Ainsi, certains chevaux sont plus aptes que les autres à fournir soit un effort bref et rapide (flèche $R$ ), soit un effort plus long (flèche L) (Figs 2 et 3 ). Une évaluation absolue des aptitudes sportives nécessiterait l'introduction soit de valeurs de références, soit de nombreux résultats obtenus dans les mêmes conditions expérimentales.

Les variables considérées à la vitesse de référence $4,65 \mathrm{~m} / \mathrm{s}$ privilégient l'étude de l'aptitude à l'endurance plutôt que la qualité de sprinter. Dans des conditions expérimentales différentes, sur une piste et non plus dans un manège couvert, les vitesses maximales obtenues à chaque palier auraient été supérieures et par conséquent la vitesse de référence aurait été plus élevée.

L'ACP effectuée décompose nettement 4 facteurs de l'aptitude sportive parmi les paramètres mesurés. En effet, chacun des 4 axes considérés recouvre des paramètres différents. II ressort que les paramètres cardio-vasculaires de l'axe 1 sont 
Tableau V. Distances de Mahalanobis entre chaque groupe de race.

\begin{tabular}{lllll}
\hline Groupe de race & G1 $=$ TF & G2 $=S F$ & G3 $=P S$ & G4 = Po \\
\hline G1 $=$ TF & 0,00 & & & \\
G2 $=$ SF & 2,998 & 0,00 & & \\
G3 $=$ PS & 3,428 & 2,515 & 0,00 & 0,00 \\
G4 = Po & 4,258 & 3,695 & 4,233 & \\
\hline
\end{tabular}

Races : TF : Trotteur Français; SF : Selle Français; PS : Pur-Sang; Po : Poney.

ceux qui différencient le mieux les chevaux de l'échantillon étudié. Viennent ensuite, par ordre d'importance décroissante, les paramètres hématocrite $(\mathrm{HT})$, lactatémie (La), fréquence des foulées (FF) et longueur des foulées (LF).

Sur les 2 premiers axes qui caractérisent la réponse du système cardiovasculaire à un effort de puissance moyenne (vitesse $=4,65 \mathrm{~m} / \mathrm{sec}$ ), les variables vitesse induisant une fréquence cardiaque de 150 bat/min $\left(V_{150}\right)$ et fréquence cardiaque à $4,65 \mathrm{~m} / \mathrm{sec}$ (FC) indiquent le niveau de travail cardiaque. Les variables PFC et PHT, qui expriment respectivement l'évolution de la fréquence cardiaque et de l'hématocrite avec la vitesse, renseignent plus précisément sur l'excitabilité neurovégétative du cheval et, par voie de conséquence, sur le niveau d'entraînement physiologique de l'organisme. Un individu de nature excitable a des valeurs PFC et PHT plus élevées (Persson, 1967). L'individu apte au sprint semble avoir une faculté de recouvrement rapide de ses fonctions cardio-vasculaires, grâce à une forte stimulation neurovégétative (système orthosympathique). L'entraînement modifie l'équilibre neurovégétatif, ce qui se traduit par une légère diminution de PFC (Persson, 1983; McArdle et al., 1985 ) et probablement de PHT. Ces 2 va- riables caractérisent mieux le fonctionnement du système cardio-vasculaire que les fréquences à des vitesses déterminées $\left(\mathrm{FC}_{\mathrm{vi}}\right)$.

L'hématocrite (HT), paradoxalement non lié à PHT, préciserait à la fois l'aptitude du cheval à mobiliser son «pool» de globules rouges spléniques et l'importance en volume de cette réserve.

Par ailleurs, sur certains chevaux de l'échantillon étudié, la relation fréquence cardiaque-vitesse, pour des vitesses $>6$ $\mathrm{m} / \mathrm{sec}$, n'est plus linéaire mais tend vers une valeur limite définissant la fréquence maximale. Pour cette raison, il a été choisi de calculer $V_{150}$ plutôt que $V_{200}$, car, pour $F C=150$, la relation est linéaire dans tous les cas, contrairement au point $F C=200$. Le problème de la surestimation de $V_{200}$ par la relation linéaire $F C$ contre vitesse a déjà été souligné par Thornton (1985).

Le facteur défini par l'axe 3 de l'ACP révèle les rapports entre le recrutement $d u$ métabolisme anaérobie et la fréquence des foulées adoptée par le cheval à la vitesse de $4,65 \mathrm{~m} \cdot \mathrm{s}^{-1}$. II semble que la fréquence des foulées (FF) explique davantage l'élévation de la lactatémie que la vitesse (vitesse $=$ FF $\times$ LF) ou la longueur des foulées (LF). Cette constatation doit être rapprochée du fait que plus le format du cheval est petit, plus la fréquence des 
foulées adoptée pour une vitesse donnée est élevée (Barrey, 1987). Ces interrelations entre paramètres d'allure et métabolisme anaérobie expliquent les lactatémies plus fortes observées chez les petites races, et notamment chez le Poney étudié.

Dans les conditions du test d'effort pratiqué, les chevaux travaillent essentiellement en aérobiose, excepté lors du dernier palier où certaines phases d'accélération ou d'efforts plus violents dans les virages nécessitent davantage de puissance et impliquent donc une participation du métabolisme anaérobie. Les valeurs de lactatémie à la vitesse de $4,65 \mathrm{~m} / \mathrm{s}$ restent donc faibles pour la majorité des chevaux $(<1 \mathrm{mmol} / \mathrm{l})$ sauf pour les individus $\mathrm{n}^{\mathrm{os}} 5$ et 6 (Tableau II).

La franche séparation des races opérée par l'AFD d'après les paramètres mesurés révèle les différences d'aptitudes sportives d'origine génétique. Ainsi, au niveau d'effort demandé, peu intense, les PurSang et les Trotteurs Français bénéficient d'une capacité aérobie supérieure aux autres races, mise en évidence par la mesure des paramètres cardio-vasculaires et lactiques. Cependant, une meilleure caractérisation des aptitudes sportives de chaque race nécessiterait une étude sur des effectifs plus importants.

\section{CONCLUSION}

L'évaluation rationnelle de l'aptitude sportive nécessite la prise en compte de nombreux facteurs qui explorent les différentes fonctions de l'organisme impliquées lors d'un effort physique. Cette étude démontre l'intérêt d'une telle approche à l'aide d'outils statistiques bien adaptés, comme l'analyse en composantes principales ou l'analyse factorielle discriminante.

Afin de mieux évaluer chaque type d'aptitude sportive, il convient d'améliorer et d'adapter la méthodologie expérimentale décrite aux différents types d'effort : endurance, résistance, sprint et saut d'obstacle.

\section{REMERCIEMENTS}

Ces travaux ont été financés par le contrat $\mathrm{Ha}$ ras Nationaux. INRA.

Les auteurs remercient vivement les responsables de la Société hippique de l'école vétérinaire d'Alfort (SHEVA) pour leur coopération lors de cette étude.

\section{RÉFÉRENCES}

Attenburrow D.P. (1983) Respiration and locomotion. In : Equine Exercise Physiology. Granta Ed., Cambridge, pp. 17-22

Barrey E. (1987) Biomécanique du pied du che$\mathrm{val}$ : étude expérimentale. Thèse vétérinaire, Ecole nationale vétérinaire d'Alfort

Barrey E. (1988) Modélisation du cheval à l'effort. Entretiens de Bichat - Tables rondes et débats, septembre 1988, Paris

Cavanagh P.R. \& Kram R. (1985) Mechanical and muscular factors affecting the efficiency of human movement. Med. Sci. Sports Exercise 17. 326-331

Dalin G. \& Jeffcott L.B. (1985) Locomotion and gait analysis. Vet. Clinics North Am. : Equine Pract. 1, 549-572

Daniels J.T. (1985) A physiologist's view of running economy. Med. Sci. Sports Exercise 17, 332-338 
Foucart T. (1985) Analyse factorielle : programmation sur micro-ordinateurs. Coll. "Méthodes Programme", Masson, Paris

Frape D.L. (1988) Dietary requirements and athletic performance of horses. Equine Vet. J. 20, 163-172

Gutteridge C.S., McFie H.J.H. \& Norris J.R. (1979) Use of principal components analysis for displaying variation between pyrograms of micro-organisms. J. Anal. Appl. Pyrolysis 1, 6676

Hörnicke H., Meixner R. \& Pollmann O. (1983) Respiration in exercising horses. In : Equine Exercise Physiology. Granta Ed., Cambridge, pp. 7-16

Hoyt D.F. \& Taylor C.R. (1981) Gait and the energetics of locomotion in horses. Nature 292, 239-240

Leach D.H. (1987) Locomotion of the athletic horse. In : Equine Exercise Physiology II. ICCEP publications, Davis, pp. 516-535

Leach D.H. \& Dagg A.I. (1983) A review on equine locomotion and biomechanics. Equine Vet. J. 15, 93-102

McArdle W.D., Katch F.I. \& Katch V.L. (1985) Exercise physiology : Energy, Nutrition and $\mathrm{Hu}$ man Performance. Lea \& Febiger, Philadelphia

McMiken (1983) An energetics basis of equine performance. Equine Vet. J. 15, 122-133

Pagan J.D. \& Hintz H.F. (1986a) Equine energetics. I. Relationship between bodyweight and energy requirements in horses. J. Anim. Sci. 63, 812-821

Pagan J.D. \& Hintz H.F. (1986b) Equine energetics. II. Energy expenditure in horses during submaximal exercise. J. Anim. Sci. 63, 822-830

Persson S.G.B. (1967) On blood volume and working capacity. Acta Vet. Scand. Suppl. 19, 1189

Persson S.G.B. (1983) Evaluation of exercise tolerance and fitness in the performance horses. In: Equine Exercise Physiology. Granta Ed., Cambridge, pp. 441-457
Persson S.G.B., Essen-Gustavsson B., Lindholm A., McMiken D. \& Thornton J.R. (1983) Cardiorespiratory and metaobolic effects of training of standardbred yearlings. In : Equine Exercise Physiology. Granta Ed., Cambridge, pp. 458-469

Pratt G.W. (1984) Racing surfaces. A survey of mechanical behavior. Proc. Am. Assoc. Equine Pract. 30, 321-331

Silver I.A., Brown P.N. \& Goodship A.E. (1983) Biochemical assessment of locomotor performance in the horse. Equine Vet. J. Suppl. 1, 2325

Thiel M., Tolkmitt G. \& Hörnicke H. (1987) Body temperature changes in horses during riding: time course and effects on heart rate and respiratory frequency. In: Equine Exercise Physiology II. ICCEP Publications, Davis, pp. 183-193

Thornton J.R. (1985) Exercise testing. Vet. Clin. North Am. : Equine Pract. 1, 573-595

Thornton J.R., Pagan J. \& Persson S. (1987) The oxygen cost of weight loading and inclined treadmill exercise in the horse. In: Equine Exercise Physiology II. ICCEP Publications, Davis, pp. 206-215

Valberg S., Essen-Gustavsson B., Lindholm A. \& Persson S. (1985) Energy metabolism in relation to skeletal muscle fibre properties during treadmill exercise. Equine Vet. J. 17, 439-444

Valette J.P. \& Wolter R. (1987) Lactate et rythme cardiaque. Critères d'aptitude sportive chez les Poneys. Congrès de chirurgie équine et médecine des sports équestres, Genève, 2528 mars 1987, pp. 70-76

Valette J.P. \& Wolter R. (1988) Intérêt des mesures de lactatémie et de fréquence cardiaque comme critères d'aptitudes sportives. CEREOPA, $14^{e}$ journée d'étude, 9 mars 1988, pp. 1-16

Wilson R.G., Isler R.B. \& Thornton J.R. (1983) Heart rate, lactic acid production and speed during a standardized exercise test in standardbred horses. In: Equine Exercise Physiology. Granta Ed., Cambridge, pp. 487-496

Wolter R. (1987) La nutrition de l'animal de sport. Sci. \& Sports 2, 69-93 